\title{
A Triad of Temporomandibular Joint Ankylosis, Mandibular Retrognathia and Severe Obstructive Sleep Apnoea

\author{
Case report
}

"Issa K. Al-Nuumani, ${ }^{1}$ Abdulaziz Bakathir, ${ }^{2}$ Ahmed Al-Hashmi, ${ }^{3}$ Mohammed Al-Abri, ${ }^{4}$ Hussein Al-Kindi, ${ }^{5}$ Intisar Al-Macki, ${ }^{6}$ Zainab Al-Balushi ${ }^{7}$

$$
\text { ثلاثية من قسط المفصل الصدغي الفكي السفلي وتراجع الفك السفلي النقطاع النفس الإنسدادي النومي الشديد }
$$

عيسى خلفان النعماني، عبدالعزيز عبداله باكثير، أحمد خميس الهاشي، محمد العبري، حسين الكندي، انتصار أل مكي، زينب البلوشية

ABSTRACT: The surgical management of paediatric patients with temporomandibular joint (TMJ) ankylosis, mandibular retrognathia and obstructive sleep apnoea (OSA) is challenging. We report a nine-year-old boy who presented to the Department of Oral Health, Sultan Qaboos University Hospital, Muscat, Oman, in 2016 with complaints of limited mouth opening, loud snoring and excessive daytime sleepiness. He was diagnosed with TMJ ankylosis, mandibular retrognathia and severe OSA. The patient initially underwent mandibular distraction and, subsequently, release of the TMJ ankylosis and rib graft reconstruction. The overall patient outcome was successful, with improvement in OSA-related symptoms, good facial symmetry and adequate mouth opening.

Keywords: Temporomandibular Joint Disorders; Temporomandibular Ankylosis; Retrognathia; Obstructive Sleep Apnea; Case Report; Oman.

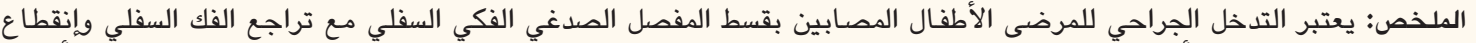

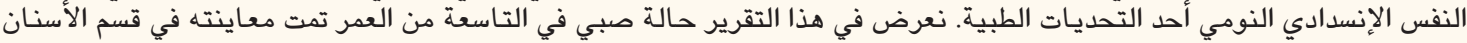

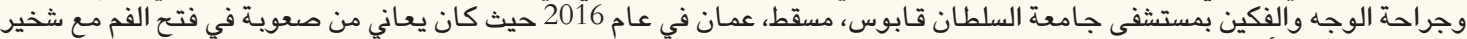

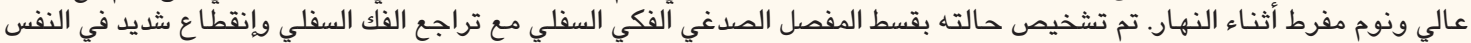

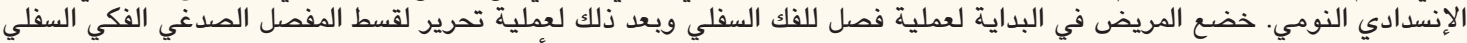

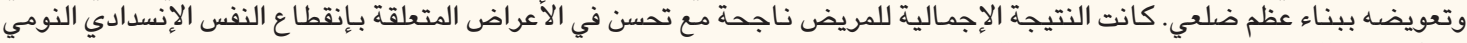

$$
\begin{aligned}
& \text { وتماثل جيد للوجه مي تحسن جيد في فتح الفم. } \\
& \text { الكلمات المفتاحية: اضطرابات المفصل الصدغي الفكي السفلي؛ قسط المفصل الصدغي الفكي السفلي؛ إنقطاع النفس الإنسدادي النومي؛ تراجع الفك } \\
& \text { السفلي؛ تقرير حالة؛ عمان. }
\end{aligned}
$$

$\mathrm{T}$ EMPOROMANDIBULAR JOINT (TMJ) ANKYLOSIS is defined as the replacement and/or fusion of the joint articular surfaces by fibrous, fibro-osseous or bony tissue resulting in the mandibular condyle becoming partially or completely joined to the skull base. ${ }^{1,2}$ There are multiple aetiological factors for TMJ ankylosis, including trauma, infections, surgical procedures and systemic diseases like rheumatoid arthritis, ankylosing spondylitis and psoriasis. ${ }^{1-3}$

In growing children, TMJ ankylosis can pose many challenges and is often associated with multiple functional and aesthetic complications such as mandibular growth retardation leading to retrognathia, micrognathia or dental malocclusion. ${ }^{3}$ Furthermore, this acquired deformity can affect airway patency, resulting in the increased risk of recurrent upper airway infections in addition to mechanical obstruction of the airway causing obstructive sleep apnoea (OSA)., ${ }^{3,4}$ This report describes a rare triad of TMJ ankylosis, mandibular retrognathia and severe OSA in a paediatric patient in which two-stage surgical management resulted in a successful outcome.

\section{Case Report}

A nine-year-old boy presented to the Department of Oral Health, Sultan Qaboos University Hospital, Muscat, Oman, in 2016 with complaints of limited mouth opening, loud snoring and excessive daytime sleepiness. His medical history was unremarkable with no reported incidents of trauma or infection. A clinical examination revealed 


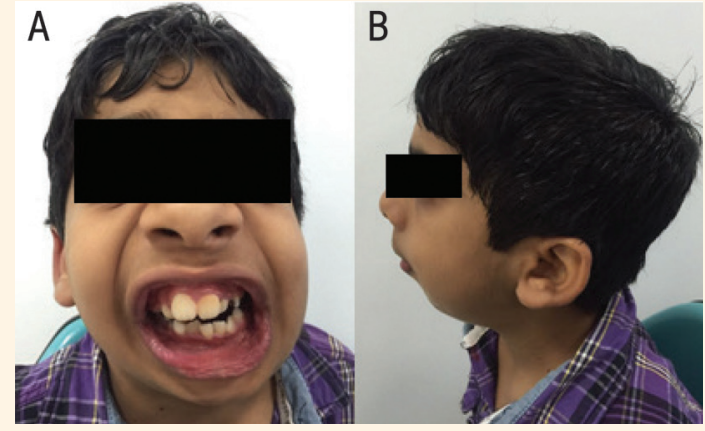

Figure 1: Preoperative photographs of a nine-yearold boy showing (A) limited mouth opening and (B) mandibular retrognathia.

limited mouth opening of $10 \mathrm{~mm}$ and mandibular retrognathia with deviation of the chin to the left side [Figure 1]. During the examination, multiple episodes of sudden sleep with rapid awakening were observed. An orthopantomogram and lateral neck imaging showed the unilateral fusion of the left condylar head to the base of the skull, indicating left-sided TMJ ankylosis and narrowing of the upper airway space [Figure 2]. Polysomnography revealed an apnoea-hypopnoea index (AHI) score of 77, indicating severe OSA. The final diagnosis was of a triad of unilateral TMJ ankylosis, mandibular retrognathia and severe OSA.

Surgical management was planned in two stages. Stage one involved a full airway assessment which showed severe narrowing of the upper airway space at the tongue base level. Subsequently, $25 \mathrm{~mm}$ bilateral mandibular distractors (Zurich Mandible Distractors ${ }^{\circledR}$, KLS Martin LP, Jacksonville, Florida, USA) were inserted to correct the retrognathia. Postoperatively, the patient was kept intubated in the intensive care unit, with the distractors activated 24 hours later and lengthened at a rate of $0.5 \mathrm{~mm}$ three times daily. It was originally planned that the patient would remain intubated until the mandible had advanced by approximately $10 \mathrm{~mm}$. However, the patient became agitated on the second postoperative day and-due to the high risk of dislodgement of the intubation tube and accidental extubation-a tracheostomy was performed on the third postoperative day in order to secure the airway. The distraction process continued until the mandible had advanced by $19.5 \mathrm{~mm}$ on the right side and $25 \mathrm{~mm}$ on the left side so as to compensate for the patient's chin deviation.

After an eight-week consolidation period, the second stage of surgical management was carried out. This involved removing the distractors, releasing the TMJ ankylosis, carrying out an ipsilateral coronoidectomy and reconstructing the condyle using a costochondral rib graft and interpositional fat graft. Intraoperatively, the mouth could be opened to $35 \mathrm{~mm}$. A postoperative orthopantomogram and lateral neck imaging indicated satisfactory opening of the upper airway [Figure 3]. The patient then began an aggressive physiotherapy regimen and was decanulated successfully on the third postoperative day.

Overall, the outcome of the two-stage surgery was successful, with notable improvements in facial symm-
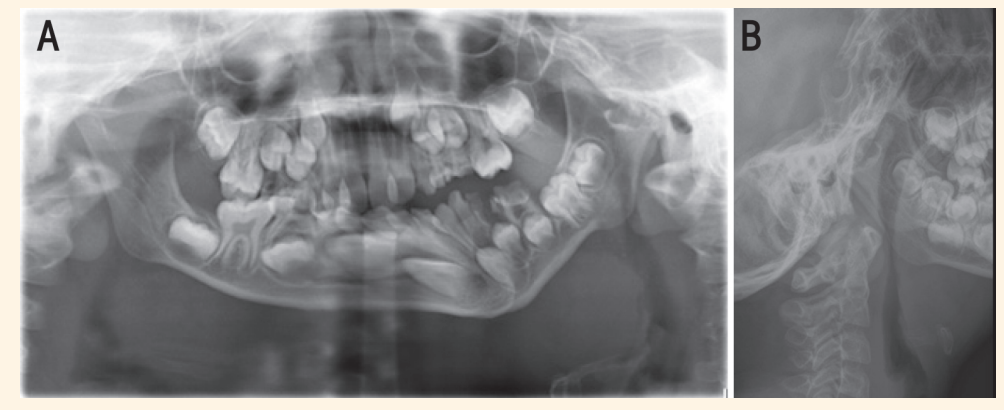

Figure 2: A: Preoperative orthopantomogram of a nine-year-old boy showing unilateral temporomandibular joint ankylosis. B: Preoperative lateral neck imaging showing obstructed airway space.
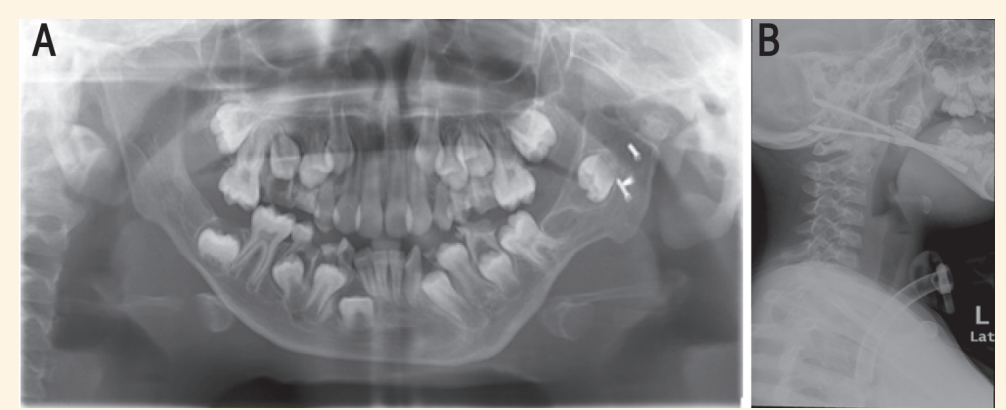

Figure 3: A: Postoperative orthopantomogram of a nine-year-old boy showing adequate joint space with a costochondral graft in situ. B: Postoperative lateral neck imaging showing adequate airway space. 


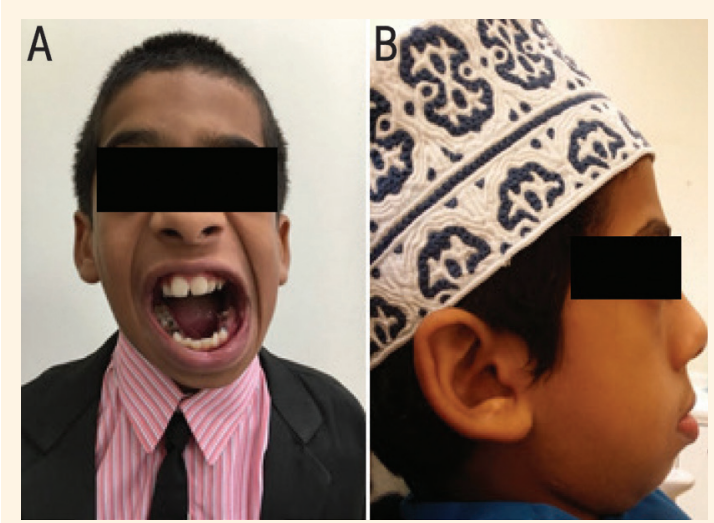

Figure 4: Postoperative photographs of a nine-year-old boy demonstrating (A) optimal mouth opening and (B) good facial symmetry. The photographs were taken a few days apart.

etry and adequate mouth opening of $40 \mathrm{~mm}$ [Figure 4]. Postoperatively, subjective feedback from the patient and his parents indicated a marked improvement in his sleeping patterns and the absence of any OSArelated sleeping disturbances, including snoring or rapid awakening. Two years later, a subsequent sleep study revealed an AHI score of 10, indicating the successful treatment of the patient's OSA.

\section{Discussion}

Ankylosis of the TMJ is a chronic disease that destroys the structure and articulation of the joint, leading to hypomobility of the jaw due to the abnormal union between the mandibular condyle and the base of the skull. ${ }^{1,2,5}$ The partial or complete replacement of the normal joint space determines the degree of hypomobility. 3,5 While there are many aetiological factors for TMJ ankylosis, trauma is the most common, followed by infections. ${ }^{1,3}$ In the current case, the aetiology of the ankylosis was unknown; this is unusual as most previous research generally reports a causative factor. ${ }^{6}$

Paediatric TMJ ankylosis is uncommon and represents a challenge in the practice of oral and maxillofacial surgery. ${ }^{3,4}$ Complicating factors include the patient's age at onset, presentation, implications regarding growth, surgical complexity, risk of recurrence and postoperative compliance. ${ }^{3-6}$ Clinical presentations of TMJ ankylosis among children include facial growth disturbances, difficulty in chewing and swallowing, poor oral health and limited mouth opening. ${ }^{7}$ Depending on the time of onset and the extent of the ankylosis, facial growth retardation may lead to mandibular retrognathia and chin deviation; the latter condition is often seen in unilateral TMJ ankylosis cases. ${ }^{7}$ Furthermore, mandibular retrognathia leads to the posterior displacement of the tongue base and is associated with a reduction in the oropharyngeal spaces, leading to upper airway reduction/obstruction and, ultimately, OSA. ${ }^{4}$ In the present case, the patient exhibited classic features of unilateral TMJ ankylosis with mandibular retrognathia and severe OSA. Among paediatric patients, the occurrence of TMJ ankylosis, mandibular retrognathia and OSA represents a rare triad of complex clinical presentations triggered primarily by TMJ ankylosis. ${ }^{1,3,4}$

Mandibular distraction osteogenesis (MDO) is increasingly used to correct mandibular retrognathia in TMJ ankylosis cases. ${ }^{3}$ In a seven-year study, Carls et al. reported successful outcomes following mandibular distraction among 14 children with retromandibulism. ${ }^{8}$ Generally, MDO has several advantages over costochondral grafting-previously considered the gold standard technique to correct this type of mandibular deformity - as the amount and direction of mandibular growth is more predictable because the callus direction can be controlled by the surgeon. ${ }^{3,9}$ Furthermore, this technique allows for the stretching of the soft tissues during the distraction period as well as the opportunity for three-dimensional augmentation with bi- or multidirectional extraoral distraction devices. Finally, the well-vascularised regenerated bone in the distraction gap obviates the need for a bone graft. ${ }^{3}$

Four different surgical protocols have been reported in the literature for managing paediatric patients with TMJ ankylosis, mandibular retrognathia and OSA. The first involves the release of the TMJ ankylosis with costochondral graft reconstruction in the hope that the graft will act as a growth centre, thus leading to the correction of the mandibular retrognathia., ${ }^{3,9}$ However, this option was not ideal in the current case because it would not immediately alleviate the patient's symptoms of severe OSA. Furthermore, this approach has been known to prolong postoperative morbidity and result in unpredictable growth. ${ }^{3,4,9}$

The second protocol involves a single-staged surgery in which ankylosis release and MDO is performed simultaneously to address both the mandibular retrognathia and OSA., ${ }^{3,10}$ Nevertheless, this choice also has significant drawbacks, including the continuous stretching of the bone during physiotherapy after the ankylosis is released, physical interference from the distractor during physiotherapy and unreliable movements of the proximal segment of the mandible after distraction. ${ }^{3,4,7,10}$ Zanaty et al. reported significant improvements in AHI and oxygen desaturation index scores and mouth opening among 30 children with micrognathia and TMJ ankylosis when using this technique; however, the protocol was modified slightly in that the ramus rather than the body of the jaw bone was targeted during MDO. ${ }^{11}$ 
The third protocol is the most popular and comprises a two-stage surgery wherein the TMJ ankylosis is released first and MDO is performed at a later stage., Nonetheless, this option is not recommended in cases of severe OSA. ${ }^{4}$ Due to the challenging nature of this condition and potential lack of cooperation during the postoperative period on the part of the paediatric patient, there is a risk of ankylosis recurrence and unpredictable regression in jaw advancement. Furthermore, published reports highlight that releasing the ankylosis first in patients with a compromised airway is usually associated with further collapse of the airway during postoperative jaw physiotherapy. ${ }^{3,4}$ Andrade et al. reported that ankylosis release alone resulted in respiratory distress during postoperative jaw exercises and a drop in oxygen saturation and heart rate among seven patients with TMJ ankylosis, micrognathia and OSA. ${ }^{4}$

The most recent protocol for the treatment of TMJ ankylosis, mandibular retrognathia and OSA was first suggested by Andrade et al. in 2012. ${ }^{4}$ In this protocol, MDO is performed initially and the ankylosis is released at a later stage in order to maintain the patency of the airway during postoperative jaw physiotherapy. ${ }^{4}$ Anantanarayanan et al. reported three paediatric cases wherein MDO was performed prior to ankylosis release. ${ }^{3}$ All three patients had favourable outcomes and were cured of snoring, with episodes of night time awakening and daytime sleepiness significantly reduced. ${ }^{3}$ The findings of the current case support utilisation of this protocol.

\section{Conclusion}

A triad of TMJ ankylosis, mandibular retrognathia and OSA is uncommon among paediatric patients. Moreover, such cases tend to be challenging, requiring careful management and early diagnosis to reduce the severity of symptoms and prevent associated complications. Although several surgical protocols exist for managing such patients, oral and maxillofacial surgeons should be aware of recent evidence supporting new approaches or modifications to existing techniques.

\section{ACKNOWLEDGEMENTS}

A poster of the preliminary version of this case report was presented at the $23^{\text {rd }}$ International Conference on Oral \& Maxillofacial Surgery from 31 March-3 April 2017 in Hong Kong. An abstract of this poster presentation was subsequently published in the International Journal of Oral \& Maxillofacial Surgery in March 2017 (Volume 46, Issue S1, P. 356).

\section{References}

1. Al-Saadi NJ, Bakathir AA, Al-Hashmi AK, Al-Ismaili MI. Temporomandibular joint ankylosis as a complication of neonatal septic arthritis: Report of two cases. Sultan Qaboos Univ Med J 2015; 15:e554-8. doi: 10.18295/squmj.2015.15.04.020.

2. Movahed R, Mercuri LG. Management of temporomandibular joint ankylosis. Oral Maxillofac Surg Clin North Am 2015; 27:27-35. doi: 10.1016/j.coms.2014.09.003.

3. Anantanarayanan P, Narayanan V, Manikandhan R, Kumar D. Primary mandibular distraction for management of nocturnal desaturations secondary to temporomandibular joint (TMJ) ankylosis. Int J Pediatr Otorhinolaryngol 2008; 72:385-9. doi: 10.1016/j. ijporl.2007.11.015.

4. Andrade NN, Kalra R, Shetye SP. New protocol to prevent TMJ reankylosis and potentially life threatening complications in triad patients. Int J Oral Maxillofac Surg 2012; 41:1495-500. doi: 10.1016/j.ijom.2012.06.012.

5. Kaban LB, Perrott DH, Fisher K. A protocol for management of temporomandibular joint ankylosis. J Oral Maxillofac Surg 1990; 48:1145-51. doi: 10.1016/0278-2391(90)90529-B.

6. Kaban LB, Bouchard C, Troulis MJ. A protocol for management of temporomandibular joint ankylosis in children. J Oral Maxillofac Surg 2009; 67:1966-78. doi: 10.1016/j.joms.2009.03.071.

7. De Roo N, Van Doorne L, Troch A, Vermeersch H, Brusselaers N. Quantifying the outcome of surgical treatment of temporomandibular joint ankylosis: A systematic review and meta-analysis. J Craniomaxillofac Surg 2016; 44:6-15. doi: 10.1016/j.jcms. 2015.08.019.

8. Carls FR, Sailer HF. Seven years clinical experience with mandibular distraction in children. J Craniomaxillofac Surg 1998; 26:197-208. doi: 10.1016/S1010-5182(98)80015-2.

9. Politis C, Fossion E, Bossuyt M. The use of costochondral grafts in arthroplasty of the temporomandibular joint. J Craniomaxillofac Surg 1987; 15:345-54. doi: 10.1016/S1010-5182(87)80081-1.

10. Papageorge MB, Apostolidis C. Simultaneous mandibular distraction and arthroplasty in a patient with temporomandibular joint ankylosis and mandibular hypoplasia. J Oral Maxillofac Surg 1999; 57:328-33. doi: 10.1016/S0278-2391(99)90683-3.

11. Zanaty O, El Metainy S, Abo Alia D, Medra A. Improvement in the airway after mandibular distraction osteogenesis surgery in children with temporomandibular joint ankylosis and mandibular hypoplasia. Paediatr Anaesth 2016; 26:399-404. doi: 10.1111/ pan.12869. 